Article

\title{
Research on Electromagnetic Radiation Characteristics of Energetic Materials
}

\author{
Yuanbo Cui ${ }^{1}$, Deren Kong ${ }^{1 *}$, Jian Jiang ${ }^{1 *}$ and Shang Gao ${ }^{1}$ \\ 1 School of Mechanical Engineering, Nanjing University of Science and Technology, Nanjing 210094, China; \\ cyb6226@njust.edu.cn (Y.C.); 217101000006@njust.edu.cn (D.K.); jiangj@njust.edu.cn (J.J); \\ shang.gao@njust.edu.cn (S.G.) \\ * Correspondence: 217101000006@njust.edu.cn; jiangj@njust.edu.cn; Tel.: +86-180-2150-8290
}

\begin{abstract}
During the explosion of energetic materials, obvious electromagnetic interference will be generated, which will affect the normal operation of surrounding electronic equipment. Experiments are still an important means to study this issue. A set of electromagnetic radiation measurement device based on short-wave omnidirectional antenna and ultra-wideband omnidirectional antenna is designed to measure the electromagnetic radiation generated by the explosion of energetic materials of different masses, and the electromagnetic radiation characteristics are obtained through data processing. The results show that the mass of the energetic material has a significant effect on the time-domain characteristics of the electromagnetic radiation generated by the explosion: the higher the mass of the energetic material, the shorter the delay response of the electromagnetic signal, the longer the duration, and the earlier the peak appears. The frequency of electromagnetic radiation signals generated by the explosion of energetic materials is mainly concentrated below $100 \mathrm{MHz}$, and the energy is most concentrated in the frequency band of $0 \sim 50 \mathrm{MHz}$. The composition of energetic materials has the greatest influence on the spectral distribution, and the spectral distribution of electromagnetic radiation produced by the explosion of explosives with different compositions has obvious specificity. The electromagnetic radiation intensity generated by the explosion of energetic materials has a strong correlation with the distance from the explosion center, and it decreases with the increase of the distance, and the decreasing range is large. The structure and detonation method of energetic materials can change the geometrical motion mode during the explosion, resulting in the non-uniformity of electromagnetic radiation propagation.
\end{abstract}

Keywords: explosion; energetic material; electromagnetic radiation; characteristic

\section{Introduction}

It produces strong electromagnetic radiation during explosion of energetic materials. Electromagnetic radiation of different intensities or frequencies can cause electromagnetic interference on nearby electronic equipment such as unmanned aerial vehicle or communication device, and in severe cases, the equipment cannot be started or even damaged, causing an accident. In order to improve the anti-electromagnetic interference performance of electronic equipment, it is necessary to measure and study the electromagnetic radiation generated by the explosion of energetic materials[1].

In 1954 Kolsky published an article in Nature on measurements of electromagnetic waves emitted from the detonation of high-explosives charges[2]. Boronin studied the physical mechanism of the electromagnetic wave generated by the explosion of condensed explosives. Boronin believed that at the initial moment of metal deformation and destruction, gaseous and solid potential energy flowed out of the formed cracks. Due to the electrokinetic effect and the friction of the potential energy on the failed shell, the shell is charged and the charged polarity of the gas and the solid are opposite. Because of the asymmetric scattering of the potential energy, the space charge of the potential energy 
of the gas and the solid forms an effective dipole. Boronin proposed that the mechanism of electromagnetic radiation produced by explosion is related to the acceleration or deceleration of certain electronic genes in the ionized air layer at the front of shock wave, which is later called the "Boronin effect" [3-4]. Boronin elaborated firstly the mechanism of electromagnetic radiation generated during the explosion of energetic materials, and guided the direction of related research in the future, which is of great significance. A.L.Kuhl explained the mechanism of electromagnetic waves generated by TNT, he believed that the movement of ionized atoms, ions, and electrons was the cause of explosive electromagnetic waves. The expansion of detonation products caused strong vibration in the surrounding air and formed a strong heat wave lasting about $20 \mu \mathrm{s}$. Temperatures as high as $11000 \mathrm{~K}$ cause significant ionization in the air, and the movement of ion plaques generates electric currents that generate electric and magnetic fields. Kuhl studied the effects of these motions through numerical simulations of TNT explosions, using high-order Godunov Equations to integrate the conservation laws of onedimensional aerodynamics, and using a very fine grid (minimum unit as $10 \mu \mathrm{m}$ ) to obtain the convergent temperature and the conductivity profile, which is used to predict the three-dimensional electromagnetic waves generated by TNT explosion[5-7]. Li Jianqiao conducted theoretical and numerical simulation studies on the natural magnetic field disturbance caused by the explosion of energetic materials, obtained the temporal and spatial distribution of the electrical conductivity and the magnetic field diffusion rate of the explosive field, and found that the explosive initiation parameters have a great influence on the magnetic field disturbance. Li Jianqiao developed a more reliable method for numerical simulation of magnetic field disturbances in explosive fields, and pointed out that when explosives are geometrically asymmetric, different magnetic field disturbances will be generated in different directions of the natural magnetic field through numerical simulations. Different types of energetic materials have different magnetic field disturbance amplitudes, but the disturbance mode is the same, and this research has not been paid attention to in related fields and has high innovative value[8-9]. Ren Huilan and Chen Hong carried out electromagnetic radiation measurement experiments on $B$ explosive and RDX-based aluminum-containing explosive respectively. The research results showed that the electromagnetic radiation signal spectrum generated by the explosion of $4.5 \sim 7.5 \mathrm{~kg}$ B explosive was mainly distributed in the range of $0 \sim 50 \mathrm{kHz}$, and the amplitude of the first pulse was basically linear to $1 / 3$ equivalent power, its arrival time is not sensitive to the amount of explosives. The intensity of electromagnetic radiation signal generated by the explosion of 160 188g RDX-based aluminum-containing explosive is in the range of $1.87 \sim 15.20 \mathrm{~V} / \mathrm{m}$, and the spectrum distribution of electromagnetic radiation is mainly concentrated in the range of $500 \mathrm{MHz}$, while the content of aluminum in the explosive has an obvious effect on the spectrum distribution of $100 \sim 500 \mathrm{MHz}[10-11]$.

In this article we use dual-frequency antenna to measure electromagnetic wave and high-speed acquisition card to record data. The electromagnetic signal is analyzed in detail using methods of noise reduction, Fourier transform, and attenuation compensation. The time-domain and frequency-domain variation characteristics of electromagnetic radiation produced by explosions are discussed, and a more comprehensive and accurate law of electromagnetic radiation has been obtained.

\section{Experimental Principle and Method}

\subsection{Experimental Principle}

Measuring electromagnetic radiation generated by explosive needs to consider factors such as electromagnetic radiation intensity, propagation law and direction, equivalent test and antenna device protection in the explosive field. According to the theory of explosion mechanics and electromagnetics, assuming $f$ is the characteristic time, $\mathrm{f}=\mathrm{t} / \mathrm{m}^{1 / 3}, \mathrm{~m}$ is the quality of explosive, the relationship between the velocity of the gas explosion product $u(f)$, the velocity of the solid particles in the explosion product $v(f)$, and the shell fragment velocity $u$ can be expressed as: 


$$
\begin{gathered}
\mathrm{u}(f)=\frac{d R(f)}{d f} \\
\mathrm{v}(f)=\mathrm{u}(0) \exp (-\mathrm{B} f)+\mathrm{B} \cdot \exp \left[-\mathrm{B} f \int_{0}^{f} u(f) e^{B f} d f\right] \\
\mathrm{u}=\frac{D}{2}\left\{\frac{1}{2 U+1}\left[1-\left(\frac{r_{0}}{r}\right)^{4}\right]\right\}^{1 / 2} \\
\mathrm{~B}=\frac{9}{5} \frac{Z}{a^{2}} m^{1 / 3}, \quad \mathrm{U}=\frac{M_{c}}{m}
\end{gathered}
$$

Where $R(f)$ is the radius of the contact surface between the gas explosion product and the shell, $\mathrm{R}(f)=1+4.6 \times 10^{4} f-0.57 \times 10^{8} f^{2}+3.3 \times 10^{10} f^{3}-10^{13} f^{4}+1.2 \times 10^{15} f^{5}$, $u(0)$ is the velocity of the gas explosion product at the initial moment. In Equation (4), $a$ is the radius of solid particle, $d$ is the particle density, and $Z$ is the viscosity coefficient. According to the characteristics of the condensed explosive, $2 \mu \mathrm{m} \leq \mathrm{a} \leq 5 \mu \mathrm{m}, \mathrm{d} \approx 2 \mathrm{~g} / \mathrm{cm}^{3}$, $\eta \approx 1.0 \times 10^{3} \mathrm{~g} /(\mathrm{cm} \cdot \mathrm{s})$, when the quality of working condition is constant, $B$ can be considered as a constant. In Equation (3), $r_{0}$ is the charging radius, $r$ is the limit expansion radius of the charging shell during explosion, Equation (3) becomes $\mathrm{u} \approx \frac{D}{2}\left(\frac{1}{2 U+1}\right)^{1 / 2}$ with ignoring $\left(\mathrm{r}_{0} / \mathrm{r}\right)^{4}$. Therefore, when studying the electromagnetic radiation of energetic materials, as long as the parameters of $m, M c, f, B$ and $D$ are considered, $u(f), v(f)$ and $u$ are considered equivalently. In electromagnetism, $\mathrm{E}=\frac{q}{4 \pi X r^{2}}$, when measuring the electromagnetic radiation of energetic materials, it just needs to consider the electric field intensity $E$, the dielectric constant of air $X$ and the distance between antenna and explosion center $r$ [1216].

\subsection{Experimental method}

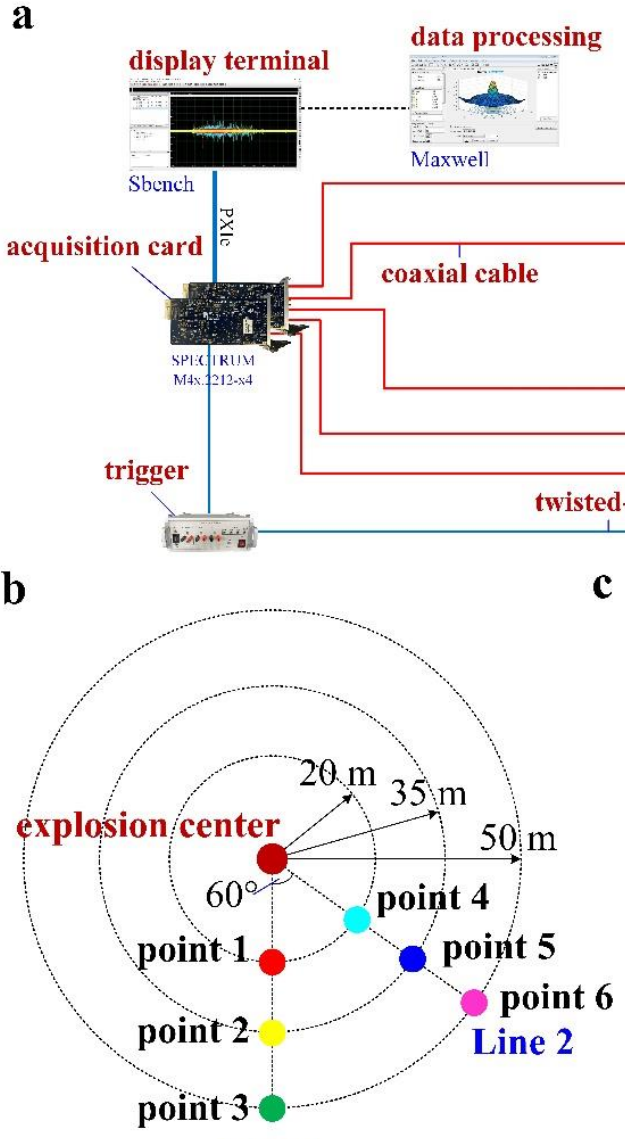

Line 1 c

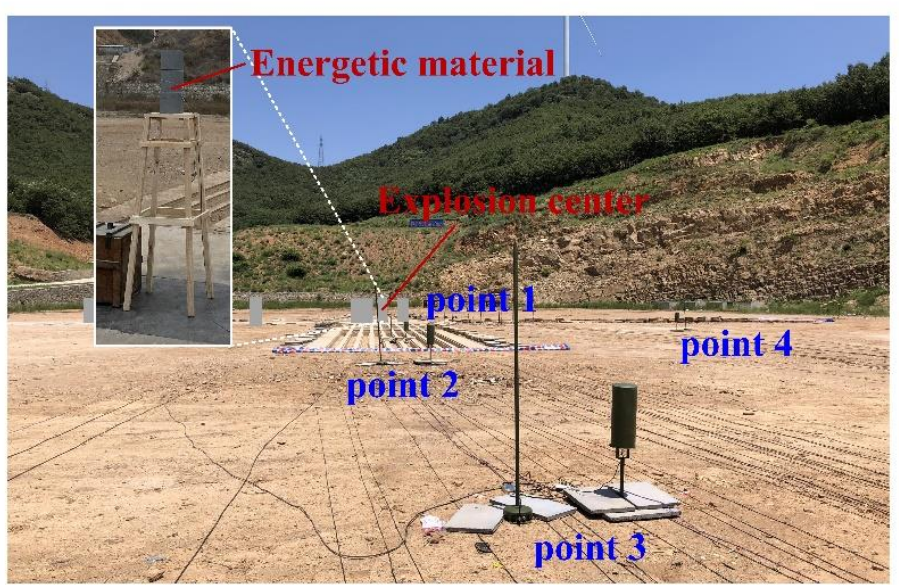

Figure 1. (a) electromagnetic radiation experimental device; (b) test points distribution; (c) experiment field.

According to the theoretical analysis of blasting mechanics, combined with the quality of explosives used in the experiment, an electromagnetic radiation measuring 
device is designed, which is shown as Figure 1(a). The test point consists of a short-wave passive omnidirectional antenna, an ultra-wideband passive omnidirectional antenna, a signal amplifier and a limiter. The short-wave passive omnidirectional antenna is a monopole antenna with the height of $2000 \mathrm{~mm}$, the sampling frequency of which is 1 $\mathrm{MHz} 30 \mathrm{MHz}$ and the output impedance is $50 \Omega$. The ultra-wideband omnidirectional antenna is a dipole antenna with the height of $450 \mathrm{~mm}$, the sampling frequency of which is $30 \mathrm{MHz} 1.5 \mathrm{GHz}$ and the output impedance is $50 \Omega$. The signal conditioner possesses multiple functions, including a combiner, signal amplifier and limiter, which can combine two electromagnetic signals of different frequencies and amplify the signal at the same time with a range of amplification factors of $10 \mathrm{~dB} \sim 30 \mathrm{~dB}$. The function of the limiter with limiting power greater than $10 \mathrm{~W}$ is to prevent the signal power from being excessively high and damaging acquisition card. The test point is connected to the data acquisition card through the radio frequency line, and the high-speed acquisition card is used to record data. The maximum sampling rate is set to $3 \mathrm{GSa} / \mathrm{s}$, the electromagnetic signal with the highest frequency of $1.5 \mathrm{GHz}$ can be collected on basis of Nyquist sampling law, which matches the frequency bandwidth of antenna. The sampling duration is set to $810 \mathrm{~ms}$, in which the sampling duration before the trigger is $10 \mathrm{~ms}$, and the sampling duration after the trigger is $800 \mathrm{~ms}[17-21]$. As shown in Figure 1(b), there are six test points in the test points distribution diagram. The point 1, point 2 and point 3 form line 1, which are respectively 20, 35 and 50 meters away from the explosion center. The point 4, point 5 and point 6 form line 2, the distance from the explosion center is 20, 35 and 50 meters respectively. The angle between Line 1 and Line 2 is 60 degrees. The measuring equipment is placed on basis of the distribution shown in Figure 1(b), and the electromagnetic radiation measurement experiment field is shown in Figure 1(c).

\section{Experimental Results and Analysis}

This paper mainly studies the electromagnetic radiation of $30 \mathrm{~kg}$ TNT and $60 \mathrm{~kg}$ TNT explosions, and the explosion experiment process of energetic material is shown in Figure 2. Before the start of the experiment, the electromagnetic background noise of the experimental site was first tested. The maximum voltage of the background electromagnetic signal measured was $62.5 \mathrm{mV}$, and the average voltage was $12.531 \mathrm{mV}$. The electromagnetic signal waveform was stable without fluctuation. It can be considered that the electromagnetic noise interference of the experimental site is extremely weak, and the explosion electromagnetic radiation experiment can be carried out.

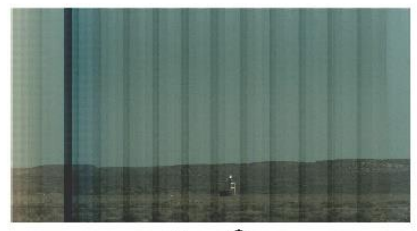

$t=0$

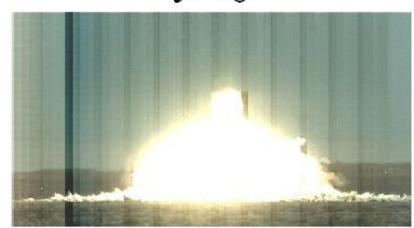

$t=0.05 \mathrm{~s}$

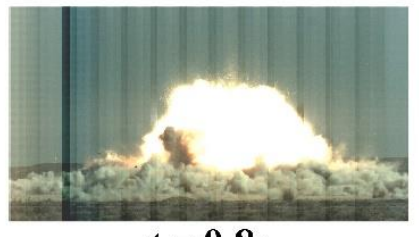

$t=0.8 \mathrm{~s}$

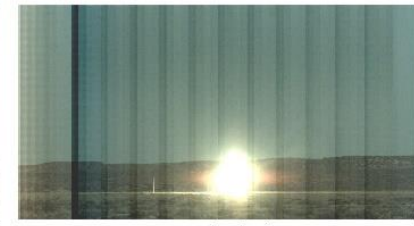

$t=0.01 \mathrm{~s}$

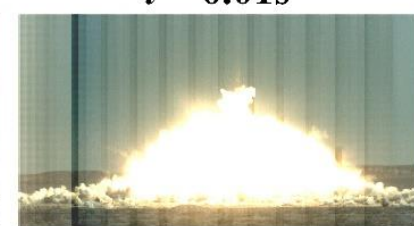

$t=0.1 \mathrm{~s}$

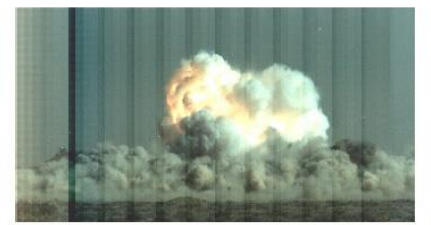

$t=1.6 \mathrm{~s}$

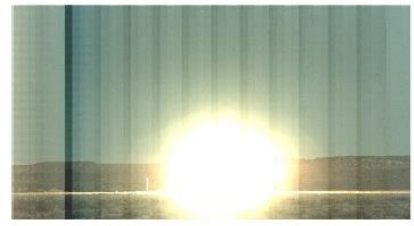

$t=0.02 \mathrm{~s}$

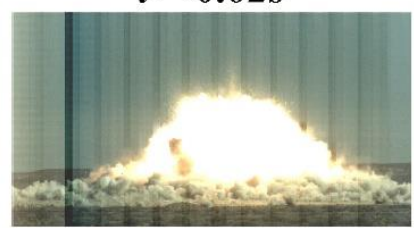

$t=0.3 \mathrm{~s}$

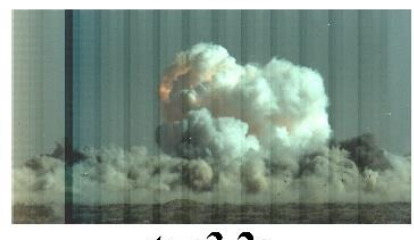

$t=3.2 \mathrm{~s}$

Figure 2. Explosion experiment process of energetic material 


\subsection{Analysis of the Time-Domain Characteristics of Electromagnetic Radiation}

The time-domain characteristics of electromagnetic radiation mainly include main parameters such as the duration in the sampling period, the delay response time, and the peak arrival time[22]. In this paper, a total of two effective experiments were carried out, and the electromagnetic signals of $30 \mathrm{~kg}$ TNT and $60 \mathrm{~kg}$ TNT explosives were measured respectively. Each group of experiments included the signals of six channels, and each channel corresponds to the test points 1 6. The electromagnetic radiation signal generated by the explosion of $60 \mathrm{~kg}$ TNT is shown in Figure 3(a), and the time-domain distribution of the electromagnetic signal of each adopted channel is shown in Figure 3(b). It can be seen from Figure 3 that the time domain distribution of the $60 \mathrm{~kg}$ TNT electromagnetic pulse is also denser, which proves that the mass of the energetic material has an important influence on the duration of the electromagnetic radiation generated by the explosion. The greater the mass, the longer the duration of the electromagnetic signal. The time-domain distribution of the electromagnetic signals measured by each test point is regular, the electromagnetic signal measured by the test points far from the explosion center has a shorter duration, and the time domain distribution of the electromagnetic pulse is also scattered.

a

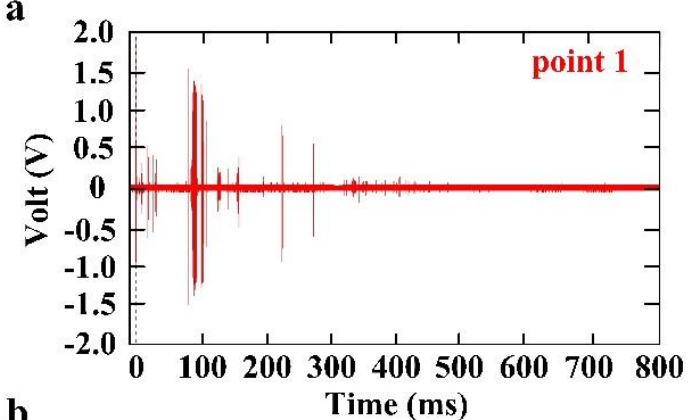

Figure 3. Electromagnetic radiation signal of TNT explosion. (a) Full-time electromagnetic radiation signal of $60 \mathrm{~kg}$ TNT explosion; (b) Time-domain distribution of electromagnetic radiation of $60 \mathrm{~kg}$ TNT explosion

The delay of the electromagnetic radiation signal generated by the explosion of $30 \mathrm{~kg}$ TNT is shown in Figure 4(a) and (b), and the delay of the electromagnetic radiation signal generated by the explosion of $60 \mathrm{~kg}$ TNT is shown in Figure 4(c) and (d). It can be seen from Figure 4 that the electromagnetic signal of $60 \mathrm{~kg}$ TNT appears significantly earlier than that of $30 \mathrm{~kg} \mathrm{TNT}$, the duration of the electromagnetic signal generated by the explosion of $60 \mathrm{~kg}$ TNT is also significantly longer than that of $30 \mathrm{~kg}$ TNT, and the peak time of the electromagnetic signal generated by the explosion of $60 \mathrm{~kg}$ TNT is significantly earlier than that of $30 \mathrm{~kg}$ TNT.

The electromagnetic radiation signal produced by the explosion of $30 \mathrm{~kg}$ TNT appeared 1.5 2.7 ms before the trigger, and a large-amplitude electromagnetic pulse appeared around $9.8 \mathrm{~ms}$. The signal delay response time of each test point is not much different, and the test point far from the explosion center has a slightly later signal appearance time. The arrival time of the electromagnetic radiation signal generated by the $60 \mathrm{~kg}$ TNT explosion is concentrated between $46 \mu \mathrm{s}$ and $62 \mu \mathrm{s}$ after the trigger time. At the 
same distance, the electromagnetic signal appearance time of the test point on the testing line 2 is $5 \sim 10 \mu$ s delayed than that of the test point on the testing line 1 , and the peak appearance time of the electromagnetic signal measured by most of the test points is between $0.31 \sim 0.39 \mathrm{~ms}$. The mass of energetic materials has a significant impact on the time-domain characteristics of the electromagnetic radiation produced by the explosion. For energetic materials of the same quality, the type and composition of explosives have no significant effect on the time-domain characteristics of electromagnetic radiation generated by the explosion.
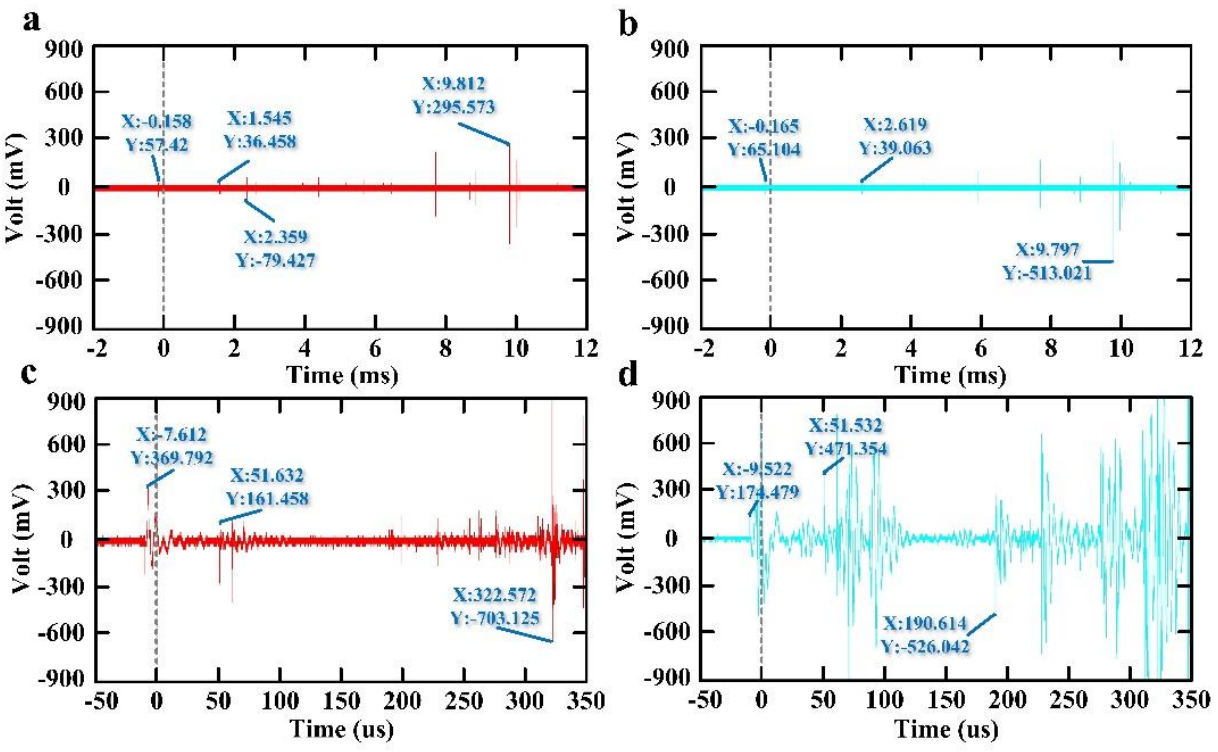

Figure 4. Delay response time of electromagnetic radiation signal of TNT explosion

\subsection{Analysis of the Frequency-Domain Characteristics of Electromagnetic Radiation}

Due to the large mass of energetic materials, the electromagnetic radiation signal lasts for a long time, and an obvious phenomenon of electromagnetic wave reflection and superposition occurs after $50 \mathrm{~ms}$. In order to facilitate comparison with previous literature data and explore the law of electromagnetic radiation of explosion, this paper extracts the electromagnetic signal within the initial $2.5 \mathrm{~ms}$, and combines the experimental results of small-equivalent energetic materials to summarize and study the law. Electromagnetic radiation signal of $60 \mathrm{~kg}$ TNT is shown in Figure 5. 

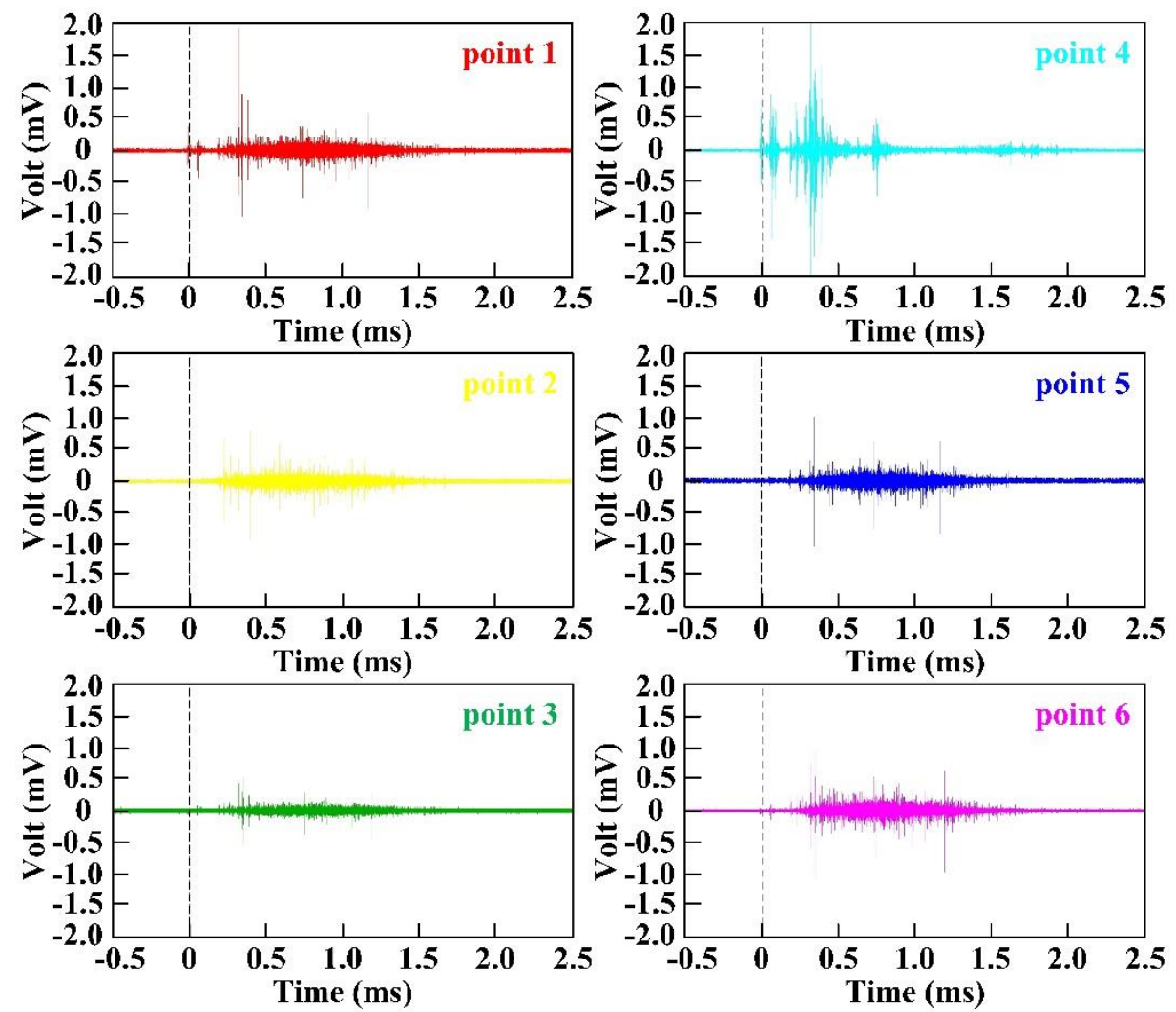

Figure 5. Electromagnetic radiation signal of $60 \mathrm{~kg}$ TNT

The spectral distribution of electromagnetic radiation is an important parameter in electromagnetic signal analysis, and the electromagnetic radiation signal is processed by means of Fourier transform with Hanning window. The electromagnetic radiation frequency distribution of $60 \mathrm{~kg}$ TNT is shown in Figure 6. The electromagnetic signal frequencies measured at test points 1 3 are mainly concentrated in $0 \sim 60 \mathrm{MHz}$ and 90 105 $\mathrm{MHz}$, and the amplitude in the $0 \sim 30 \mathrm{MHz}$ frequency band is larger. The electromagnetic signal frequencies measured at test point 4 are mainly concentrated in $0 \sim 100 \mathrm{MHz}$, and the amplitude is larger in the $0 \sim 40 \mathrm{MHz}$ frequency band. The frequency of electromagnetic signals measured at test point 5 is mainly concentrated in $0 \sim 40 \mathrm{MHz}$, of which the amplitude is larger in the $0 \sim 20 \mathrm{MHz}$ frequency band, and there is a small amount of signal distribution in the 90 100 MHz frequency band. The electromagnetic signal frequencies measured at test point 6 are mainly concentrated in $0 \sim 20 \mathrm{MHz}$ and $80 \sim 100 \mathrm{MHz}$, and the amplitude is larger in the $0 \sim 10 \mathrm{MHz}$ frequency band. 

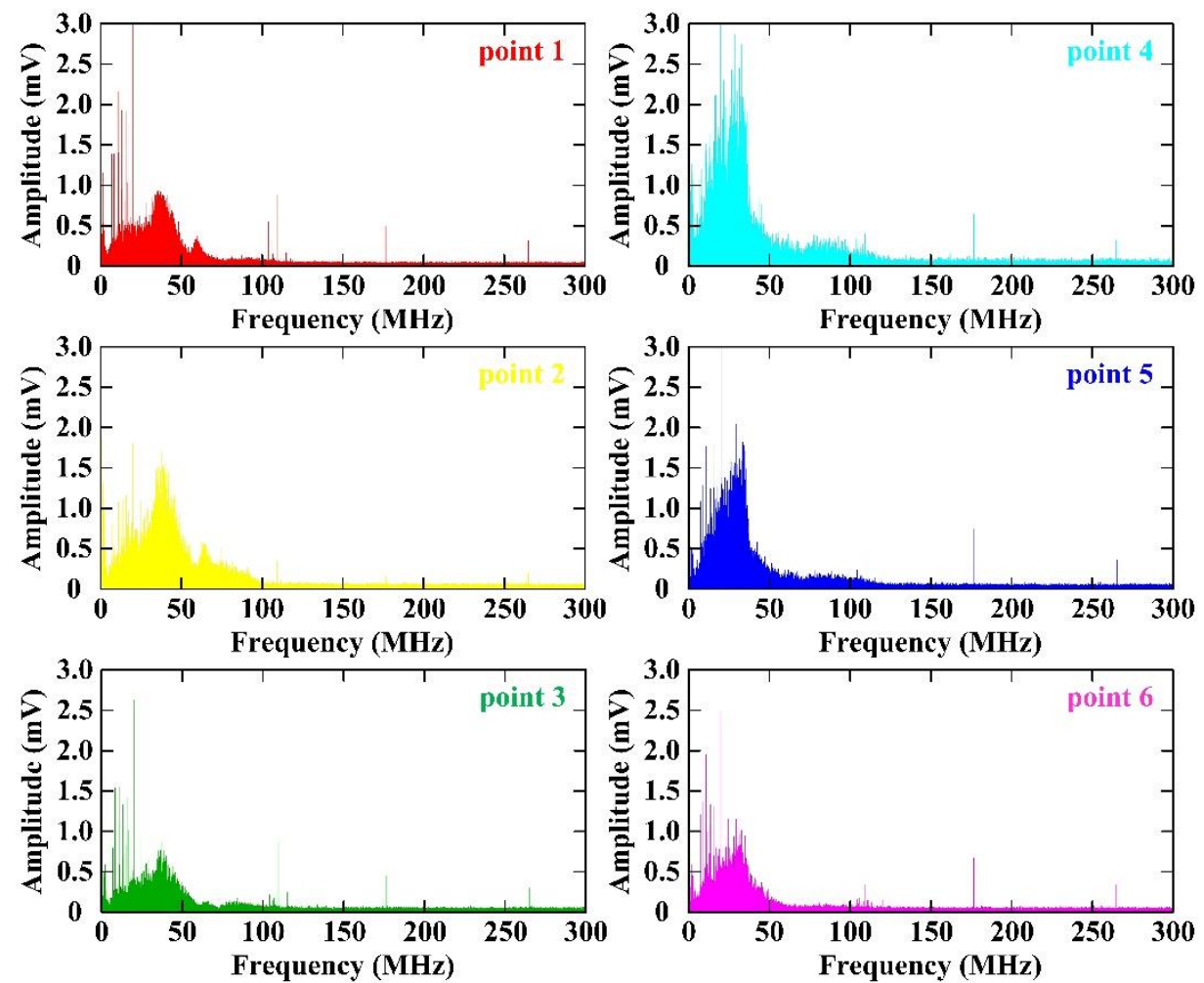

Figure 6. Spectrum of electromagnetic radiation signal of $60 \mathrm{~kg}$ TNT

According to the experimental data in the references, it can be concluded that the larger the mass of the energetic material, the larger the frequency distribution range of the electromagnetic radiation signal generated by the explosion, and the more concentrated the energy. The composition of energetic materials has the greatest influence on the spectrum distribution, and the electromagnetic radiation spectrum distribution produced by the explosion of energetic materials with different compositions has obvious specificity. This conclusion can be applied to the identification and identification of explosive composition. The closer the distance to the explosion center, the larger the frequency distribution range of the electromagnetic signal, and the electromagnetic frequency distribution in different directions is obviously different[23-25].

\subsection{Analysis of the Energy-Domain Characteristics of Electromagnetic Radiation}

Electromagnetic radio frequency radiation hazards mainly include protection location, spectrum characteristics, power intensity, signal time domain characteristics, and protection requirements. The environmental analysis of transient electromagnetic hazards mainly includes protection location, frequency band, and intensity. Electromagnetic strength is the most important reference value for electromagnetic hazard protection[26]. The energetic materials used in this experiment are of relatively high quality, and the energy produced by the explosion is relatively strong. The data acquisition equipment is placed in a shelter for protection, and the distance between the data acquisition equipment and the front-end sensor (antenna) is relatively long. In the experiment, the SYV50-5-1 coaxial cable is used for incoming signal transmission. The total length of the coaxial cable is $100 \mathrm{~m}$. As the signal frequency increases, the attenuation rate of the electromagnetic signal will gradually increase. Therefore, it is necessary to perform attenuation compensation and gain correction in the experimental data processing, and also consider factors such as antenna coefficient, antenna gain, signal conditioner gain and adapter loss. The correction parameters of this experimental device are shown in Figure 7. 


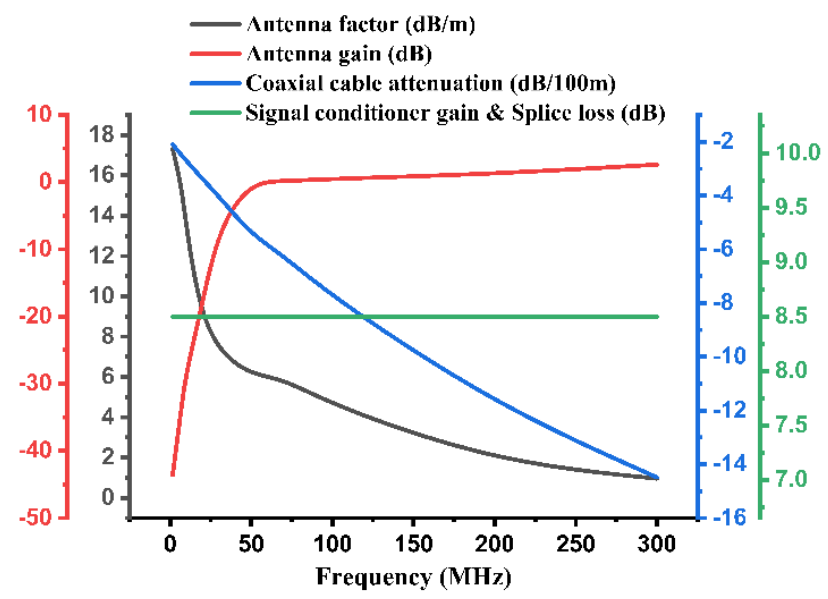

Figure 7. Experimental data correction parameters

The output of the electromagnetic sensor (antenna) of the electromagnetic measurement system is the voltage value, and the voltage value can be converted into the electric field intensity $\mathrm{E}(\mathrm{V} / \mathrm{m})$ by using the antenna coefficient and the equivalent power value. The output voltage of the measuring antenna is $U(V)$, the antenna gain after calibration is $G(d B)$, and the antenna coefficient is $A F(d B / m)$. The space energy flux density is shown in Equation 5, the effective area of the antenna is shown in Equation 6, and the antenna received power is shown in Equation 7.

$$
\begin{gathered}
\mathrm{S}=\left(\frac{1}{2} E^{2}\right) / 120 \pi \\
\mathrm{A}=\mathrm{G} \lambda^{2} / 4 \pi \\
\mathrm{P}=\mathrm{S} \cdot \mathrm{A}=U^{2} / 2 Z_{0}
\end{gathered}
$$

In the formulas, $Z_{0}$ is the system impedance $\left(Z_{0}=50 \Omega\right)$, the propagation speed of electromagnetic wave in vacuum is the speed of light $\left(c=3.0 \times 10^{8} \mathrm{~m} / \mathrm{s}\right)$. The relationship between electromagnetic frequency $f(\mathrm{~Hz})$ and wavelength $\lambda(m)$ is $f \lambda=c$. According to these formulas, Equation 8 can be obtained, where the unit of $f_{M}$ is $M H z$. The antenna factor $A F$ is as in Equation 9. Substituting $A F(d B)$ into Equation 9 yields Equation 10. Finally, the conversion relationship between signal power $P(\mathrm{dBm})$ and electromagnetic strength $E(d B \mu V / m)$ is obtained as Equation 11.

$$
\begin{gathered}
\mathrm{U}=\frac{96.82}{\pi} \mathrm{E} \sqrt{G} \frac{1}{f_{M}} \\
\mathrm{AF}=\frac{E}{U}=\frac{\pi f_{M}}{96.82 \sqrt{G}} \\
\mathrm{AF}=20 \log f_{M}-10 \log G-29.78 \\
\mathrm{P}=\mathrm{E}-\mathrm{AF}-107
\end{gathered}
$$

The experimental results after data correction are shown in Table 1. The maximum value of electromagnetic radiation intensity produced by $30 \mathrm{~kg}$ TNT explosion is 85.56 $\mathrm{V} / \mathrm{m}$, and the maximum value of electromagnetic radiation intensity produced by $60 \mathrm{~kg}$ TNT explosion is $168.86 \mathrm{~V} / \mathrm{m}$. The electromagnetic radiation intensity of $60 \mathrm{~kg}$ TNT measured at the same test point is $96.2 \% \sim 304.3 \%$ higher than that of $30 \mathrm{~kg}$ TNT. The electromagnetic radiation intensity produced by the explosion of explosives with different masses differs by more than one time. For the electromagnetic radiation intensity produced by the explosion of explosives of the same quality, the electromagnetic intensity decreases with the increase of the distance from the detonation center. For test points at the same distance and in different directions, there are also differences in the measured electromagnetic radiation intensity. The electromagnetic radiation intensity measured at the test points of $30 \mathrm{~kg}$ TNT in different directions is quite different, and the difference range is $17.35 \% \sim 102.17 \%$. The electromagnetic radiation intensity measured by $60 \mathrm{~kg}$ TNT in different directions has a small difference, and the difference range is $11.1 \% \sim 17.7 \%$.

Table 1. Experimental data of electromagnetic radiation of TNT explosion 


\begin{tabular}{ccccccccc}
\hline \multirow{2}{*}{$\begin{array}{c}\text { Test } \\
\text { point }\end{array}$} & \multicolumn{2}{c}{ Peak voltage / V } & \multicolumn{2}{c}{ Effective voltage / V } & \multicolumn{2}{c}{ Signal power / dBm } & \multicolumn{2}{c}{$\begin{array}{c}\text { Electromagnetic } \\
\text { intensity / } \cdot \mathbf{m}^{-1}\end{array}$} \\
\cline { 2 - 9 } & $30 \mathrm{~kg}$ & $60 \mathrm{~kg}$ & $30 \mathrm{~kg}$ & $60 \mathrm{~kg}$ & $30 \mathrm{~kg}$ & $60 \mathrm{~kg}$ & $30 \mathrm{~kg}$ & $60 \mathrm{~kg}$ \\
\hline Point 1 & 1.426 & 1.914 & 11.408 & 20.468 & 34.154 & 39.231 & 85.56 & 168.86 \\
Point 2 & 0.644 & 1.563 & 7.187 & 15.841 & 30.141 & 37.005 & 57.49 & 130.68 \\
Point 3 & 0.703 & 0.898 & 2.371 & 9.181 & 20.508 & 32.268 & 18.73 & 75.74 \\
Point 4 & 0.507 & 2.500 & 5.161 & 18.881 & 27.264 & 38.530 & 42.32 & 151.99 \\
Point 5 & 0.683 & 1.875 & 3.829 & 14.168 & 24.672 & 36.036 & 29.29 & 114.05 \\
Point 6 & 0.332 & 0.585 & 2.152 & 4.214 & 19.667 & 25.504 & 15.96 & 64.33 \\
\hline
\end{tabular}

\section{Conclusion}

Aiming at the phenomenon of electromagnetic radiation generated by the explosion of energetic materials, this paper carried out the electromagnetic radiation measurement experiment of energetic materials explosion. According to the analysis of relevant literature, the following main conclusions were obtained:

(1) The time-domain characteristics of the electromagnetic radiation generated by the explosion of energetic materials are most affected by the quality of the explosive. For energetic materials of the same mass, the time-domain distribution of electromagnetic radiation measured at different test points is roughly the same, but the farther away from the explosion center, the shorter the duration of electromagnetic radiation. Additionally, there are some differences in the time-domain distribution of electromagnetic radiation measured at test points in different directions.

(2) The frequency of electromagnetic radiation signals generated by the explosion of energetic materials is mainly concentrated below $100 \mathrm{MHz}$. The greater the quality of energetic materials, the wider the frequency distribution of electromagnetic radiation and the more concentrated the energy. The composition of energetic materials has a great influence on the spectrum distribution. The electromagnetic radiation spectrum distribution produced by the explosion of energetic materials with different compositions has obvious specificity, which can be applied to the identification and identification of explosive components.

(3) The electromagnetic radiation generated by the explosion of energetic materials can last until $600 \mathrm{~ms}$ after the explosion, the electromagnetic pulse is mainly concentrated in $0 \sim 300 \mathrm{~ms}$, and the energy is most concentrated in the period of 80 110 ms. During the explosion of energetic materials with different masses, the first electromagnetic radiation signals appear at different times, but they are all concentrated within $100 \mu$ s.

(4) For the electromagnetic radiation intensity produced by the explosion of energetic materials of the same mass, the intensity decreases greatly with the increase of distance. There is a large difference in the intensity of electromagnetic radiation at the same distance but in different directions. The configuration of the charge and the way of detonation make the geometric movement pattern of the explosive change during the explosion process, resulting in non-uniformity in the propagation of electromagnetic radiation.

Author Contributions: Conceptualization, Y.C.; methodology, Y.C.; formal analysis, Y.C. and S.G.; investigation, Y.C. and S.G.; data curation, Y.C. and S.G.; resources, D.K. and J.J.; writing-original draft preparation, Y.C.; writing-review and editing D.K. and S.G.; supervision, D.K. and J.J.; project administration, D.K. and J.J.; funding acquisition, D.K. and J.J. All authors have read and agreed to the published version of the manuscript.

Funding: This research was funded by the Fundamental Research Funds for the Central Universities under Grant 309181A8804 and Grant 30919011263, in part by the Natural Science Foundation of Jiangsu Province, China under Grant BK20190464, in part by the Jiangsu Planned Projects for 
Postdoctoral Research Funds under Grant 1003-YBA20012, in part by the Chinese Postdoctoral Science Foundation under Grant 2020M671481.

Institution Review Board Statement: Not applicable.

Informed Consent Statement: Not applicable.

Conflicts of Interest: The authors declare no conflict interest.

\section{Reference}

1. Cui, Y.; Kong, D. Analysis of electromagnetic radiation spectrum during the explosion of energetic materials. 2020 IOP Conf. Ser.: Earth Environ. Sci. 2020, 585, 012026.

2. Kolsky, H. Electromagnetic waves emitted on detonation of explosives. Nature 1954, 173(4393), 77-77.

3. Boronin, A.; Vel Min, V.; Medvedev, Y. et al. Experimental study of the electromagnetic field in the near zone of explosions produced by solid explosives. Journal of Applied Mechanics and Technical Physics 1968, 9, 712-717.

4. Boronin, A.; Kapinos, V.; Krenev, S. et al. Physical mechanism of electromagnetic field generation during the explosion of condensed explosive charges. Combustion Explosion and Shock Waves 1990, 26, 597-602.

5. Kuhl, A.; Bell, J.; Beckner, V. Heterogeneous continuum model of aluminum particle combustion in explosions. Combustion, Explosion, and Shock Waves 2010, 46(4), 433-448.

6. Kuhl, A.; Bell, J.; Beckner, V. et al. Spherical combustion clouds in explosions. Shock Waves 2013, 23(3), $233-249$.

7. Kuhl, A.; White, D.; Kirkendall, B. Electromagnetic waves from TNT explosions. Journal of Electromagnetic Analysis \& Applications 2014, 6(10), 280-295.

8. Li, J.; Song, W.; Ning, J. Theoretical and numerical predictions of hypervelocity impact-generated plasma. Phys Plasmas 2014, $21,082112$.

9. Li, J.; Hao, L.; Li, J. Theoretical modeling and numerical simulations of plasmas generated by shock waves. Sci. China Technol. Sci. 2019, 62, 2204-2212.

10. Ren, H.; Chu, Z.; Li, J. Study on electromagnetic radiation generated during detonation. Propellants Explosives Pyrotechnics 2019, 44(12), 1541-1553.

11. Chen, H.; Pan, X.; He, Y. et al. Measurement of time-varying electron density of the plasma generated from a small-size cylindrical RDX explosion by Rayleigh microwave scattering. Plasma Science and Technology 2021, 23(4), 045401(12).

12. Van Lint, V. Electromagnetic emission from chemical explosions. IEEE T Nucl. Sci. 1982, 29,1843-1849.

13. Soloviev, S. Generation of electric and magnetic field during detonation of high explosive charges in boreholes. J. Geophys. Res. Solid Earth 2005, 110, 1-14.

14. Dai, Q.; He, J.; Wang, S.; Li, C. Experimental study on wideband electromagnetic radiation from plasma cloud, High Power Laser Part Beams 2010, 22, 1399-1403.

15. Cao, J.; Xie, S.; Su, D.; Ma, Z. The experimental research on the electromagnetic radiation aroused by the detonation of explosive in the close space. J. B. Univ. Aeronaut Astronaut 2011, 37, 1384-1387.

16. Wang, C.; Zhou, G.; Cai, Z. et al. Measurement and analysis of shock wave overpressure of thermal explosion of charge with shell. Acta. Armamentarii. 2012, 33, 574-578.

17. Cui, Y.; Shang, F.; Kong, D.; Wang, L. Research on testing technology of electromagnetic radiation characteristics in explosive field. Initiator \& Pyrotechnics 2019, 5, 1-5.

18. Cui, Y.; Jiang, J.; Kong, D.; Gao, S.; Wang, S. Study on electromagnetic radiation interference caused by rocket fuel. Sensors 2021, 21, 8123.

19. Gao, S.; Tian, G.; Dai, X. et al. A lightweight wireless overpressure node based efficient monitoring for shock waves. IEEE/ASME Transactions on Mechatronics 2021, 26(1), 448-457.

20. Gao, S.; Lin, Y.; Zhu, J. The effect of mounting structure and piezoelectric pressure probe sensor incident angle on the free-field measurement. IEEE Sensors Journal 2019, 19(17), 7226-7233.

21. Gao, S.; Tian, G.; Dai, X. et al. A novel distributed Linear-Spatial-Array sensing system based on multichannel LPWAN for Large-Scale blast wave monitoring. IEEE Internet of Things Journal 2019, 6(6), 9679-9688.

22. Cui, Y.; Kong, D. Analysis of electromagnetic radiation spectrum of a certain type of bomb during static explosion. Initiator \& Pyrotechnics 2020, 5, 18-22.

23. Tasker, D.; Whitley, V.; Lee, R. et al. Electromagnetic field effects in explosives. American Institute of Physics 2009, 1195, 335338.

24. Wang, C.; Liu, X.; Li, X. et al. The experimental research on the electromagnetic radiation aroused by detonation of explosive. Acta. Armamentarii. 2014, 35, 188-192.

25. Tang, E.; Tang, W.; Xiang, S. et al. Coil measurement system for weak magnetic field generated by hypervelocity impact. High Power Laser Part Beams 2010, 22, 1132-1136.

26. Cui, Y.; Kong, D.; Zhang, X.; Wang, L. Measurement and analysis of electromagnetic radiation signals of TNT explosion. Chinese Journal of Energetic Materials 2021, 29(3), 241-250. 